\title{
Application of network construction to estimate functional changes to insulin receptor substrates 1 and 2 in Huh7 cells following infection with the hepatitis $\mathbf{C}$ virus
}

\author{
JINGKUN LIU ${ }^{1}$, LINBANG WANG ${ }^{2}$, WENJUN WANG ${ }^{1}$, YAPING LI $^{1}$, XIAOLI JIA ${ }^{1}$, \\ SONG ZHAI ${ }^{1}$, JUAN SHI ${ }^{1}$ and SHUANGSUO DANG ${ }^{1}$
}

${ }^{1}$ Department of Infectious Diseases, The Second Affiliated Hospital of Medical School of Xi'an Jiaotong University, Xi'an, Shaanxi 710004; ${ }^{2}$ The First Clinical Department, Chongqing Medical University, Chongqing 400016, P.R. China

Received July 8, 2015; Accepted May 3, 2016

DOI: $10.3892 / \mathrm{mmr} .2016 .5527$

\begin{abstract}
Hepatitis C virus (HCV) is closely associated with insulin resistance (IS), acting primarily by interfering with insulin signaling pathways, increasing cytokine-mediated (tumor necrosis factor $\alpha$, interleukin 6 ) inflammatory responses and enhancing oxidative stress. In the insulin signaling pathways, the insulin receptor substrate (IRS) is one of the key regulatory factors. The present study constructed gene regulatory sub-networks specific for IRS1 and IRS2 in Huh7 cells and HCV-infected Huh7 (HCV-Huh7) cells using linear programming and a decomposition algorithm, and investigated the possible mechanisms underlying the function of IRS1/2 in $\mathrm{HCV}$-induced IS in Huh7 cells. All data were obtained from GSE20948 of the Gene Expression Omnibus database from the National Center for Biotechnology Information. Genes which were significantly differentially expressed between Huh7 and HCV-Huh7 cells were analyzed using the significance analysis of microarray algorithm. The top 50 genes, including IRS1/2, were used as target genes to determine the gene regulatory networks and next the sub-networks of IRS1 and IRS2 in HCV-Huh7 and Huh7 cells using Gene Regulatory Network Inference Tool, an algorithm based on linear programming and the decomposition process. The IRS1/2 sub-networks were divided into upstream/downstream groups and activation/suppression clusters, and were further analyzed using Molecule Annotation System 3.0 and Database for Annotation, Visualization, and Integrated Discovery software, two online platforms for enrichment and clustering analysis and visualization. The
\end{abstract}

Correspondence to: Professor Shuangsuo Dang, Department of Infectious Diseases, The Second Affiliated Hospital of Medical School of Xi'an Jiaotong University, 157 Xi'wu Road, Xi'an, Shaanxi 710004, P.R. China

E-mail: dang212@126.com

Key words: hepatitis C virus, Huh7 cells, insulin receptor substrate 1 , insulin receptor substrate 2, insulin resistance, gene network reconstruction tool, GRNInfer results indicated that in Huh7 cells, the downstream network of IRS2 is more complex than that of IRS1, indicating that the insulin metabolism in Huh7 cells may be primarily mediated by IRS2. In HCV-Huh7 cells, the downstream pathway of IRS2 is blocked, suggesting that this may be the underlying mechanism in HCV infection that leads to insulin resistance. The present findings add a further dimension to the understanding of the pathological mechanisms of $\mathrm{HCV}$ infection-associated insulin resistance, and provide novel concepts for insulin resistance and glucose metabolism research.

\section{Introduction}

The occurrence and development of insulin resistance (IS) are complex processes involving the mutual regulation of numerous gene networks. Previous studies have indicated that hepatitis $\mathrm{C}$ virus (HCV) infection promotes hepatic IS, which is closely associated with the dysfunction of insulin receptor substrate (IRS) pathways, which are important in the glucose metabolism (1-3). Microarray data processing facilitates the construction of cellular gene regulatory networks and clarifies the possible interactions among genes. Further analysis of these networks confirms that certain biological processes or signaling pathways involve multiple genes and aids in the deduction of the associations between biological molecules including action consistency, metabolism direction and co-expression association (4).

In the present study, the networks of 50 major differentially expressed genes between Huh7 and HCV-Huh7 cells were constructed using the Gene Regulatory Network Inference Tool (GRNInfer) linear analysis software. The upstream and downstream networks of each gene were confirmed and were extrapolated to identify whether they are enhanced or inhibited. Additionally, the function alterations of IRS1 and IRS2 at gene network level in Huh7 and HCV-Huh7 cells were projected using Molecule Annotation System (MAS) version 3.0 online gene annotation and cluster software and the Database for Annotation, Visualization, and Integrated Discovery (DAVID). These networks were used to determine the impacts of HCV infection on the metabolism of Huh7 cells and the relevant signaling mechanisms. 


\section{Materials and methods}

Data sources. The data used in the current study were obtained from the Gene Expression Omnibus (GEO) expression database from the National Center for Biotechnology Information (www.ncbi.nlm.nih.gov/geo/). The microarray serial number used in the current study used is GSE20948 (www.ncbi.nlm. nih.gov/geo/query/acc.cgi?acc=GSE20948). The array contains 14 groups of Huh7 cells and 14 groups of HCV-Huh7 cells, each including 2 groups of cells extracted at $6 \mathrm{~h}, 3$ groups at $12 \mathrm{~h}, 3$ groups at $18 \mathrm{~h}, 3$ groups at $24 \mathrm{~h}$ and 3 groups at $48 \mathrm{~h}$. The raw data were collected in the CEL format, standardized and converted into a logarithm using Expression Console 1.4.1, 64-bit software from Affymetrix, Inc. (Santa Clara, CA, USA).

Significance analysis of gene expression. Significance analysis of microarray (SAM) is a t-test-based statistical analysis method used to determine significant differences when using microarray data. The present study used the SAM function of MultiExperiment Viewer sub-software of TM4 (www.tm4.org/mev.html) to analyze the alterations in gene functions and select the top 50 significantly differentially expressed genes (Table I).

Construction of generegulatorynetworks. The gene networks of the top 50 significantly differentially expressed genes between Huh7 and HCV-Huh7 cells were constructed using GRNInfer (digbio.missouri.edu/grninfer/) and GVedit version 2.38 (portableapps.com/node/38245) tools. GRNInfer (5) is a linear programming and decomposition algorithm for calculating gene networks from microarray data. Gene regulation is frequently non-linear; however, due to the complexity of biological structures and lack of data, various gene regulatory networks available are based on linear or additive models. From the perspective of dynamic systems, linear equations may be used to elucidate the main features of networks or functions. The following equation may represent every possible network of a data set (5): $\mathrm{J}=\left(\mathrm{X}^{\prime}-\mathrm{A}\right) \mathrm{U}^{\wedge 1} \mathrm{~V}^{\mathrm{T}}=\mathrm{YV}=\mathrm{J}+\mathrm{YV}^{\mathrm{T}}$. Where $J=\left(J_{i j}\right) m \times m=\delta \delta(\chi) / \delta \chi$ is an $n \times m$ Jacobian matrix or connectivity matrix, $\mathrm{X}=\left[\chi\left(t_{1}\right), \ldots, \chi\left(t_{\mathrm{m}}\right)\right], \mathrm{J}$ and all $m \mathrm{x} n$ matrices with $\chi_{i}^{\prime}\left(t_{j}\right)=\left[\chi_{i}\left(t_{j}+1\right)-\chi_{i}\left(t_{j}\right)\right] /\left(t_{j}+1-t_{j}\right)$ for $i=1, \ldots, \mathrm{n}$; $j=1, \ldots \mathrm{m} . X(t)=\chi_{1}(t), \ldots, \chi \mathrm{n}(t)^{\mathrm{T}} \mathrm{E} R^{\mathrm{n}}, a=\left(a_{1}, \ldots, a_{\mathrm{n}}\right)^{\mathrm{T}} \mathrm{E} R n, \chi i(t)$ is the expression level (mRNA concentrations) of gene $i$ at time intance $t . y=\left(y_{i j}\right)$ is an $n \times m$ matrix, where $y_{i j}$ is zero if $\mathrm{e}^{j}=0$. $U$ is a unitary $m \times n$ matrix of left eigenvectors, ${ }^{\wedge}=\operatorname{diag}\left(e_{1}, \ldots, e_{n}\right)$ is a diagonal $n \times m$ matrix containing $n$ eigenvalues and $V^{\mathrm{T}}$ is the transpose of a unitary $n \times m$ matrix of right eigenvectors (5). The parameters selected were $\lambda=0.0$ and threshold $=1 \times 10^{-9}$.

DAVID cluster analysis. DAVID (david.ncifcrf.gov/) is a gene function clustering tool using the bio-module as the center for large-scale genome analysis (6,7). It combines Kappa statistics features and the heuristic fuzzy clustering features and converts the model centered on functional annotation terminology and gene functions into a biological block pattern, extracting gene function annotation data from different biological databases and enriching common functional annotation of these databases.

MAS 3.0 analysis. MAS 3.0 (bioinfo.capitalbio.com/mas3/) is a free online analysis platform for high-throughout microarray gene function annotation andenrichment analysis. Its annotation system utilizes the following databases: Genbank, European Molecular Biology Laboratory, SwissPort, Gene Ontology (GO), Kyoto Encyclopedia of Genes and Genomes (KEGG), BioCarta, GeneMAPP, mirBase, Eukaryotic Promoter, Human Protein Reference Database, Membrane-Based Interactome Database, Biomolecular Interaction Network Database, Intact, TRANScription FACtor, UniGene, Single Nucleotide Polymorphism Database, Online Mendelian Inheritance in Man, InterPro, Human Genome Organisation, Mouse Genome Informatics and the Rat Genome Database, in order to provide functional annotations of genes, mRNAs, proteins, GO, metabolic pathways, regulatory genes, diseases, small interfering RNAs and tissue factors. The MAS 3.0 system provides flexible and interactive enrichment features. Using enrichment analysis with the pathway as the index as an example, the system can provide the index by input symbol, index by pathway and gene correlation as the three possible enrichment paths. The index by pathway system provides the pathway enrichment results of the three databases KEGG, GeneMAPP and BioCarta and presents the results in data table and gene-pathway network graph forms.

\section{Results}

Construction of IRSI and IRS2 networks in Huh7 and $\mathrm{HCV}$-Huh7 cells. From the 50 significantly differentially expressed genes in Huh7 and HCV-Huh7 cells, IRS1 (fold change, 4.919549) and IRS2 (fold change, 5.273203) alone belong to the IRS family. Therefore, they were used as the target genes for further analysis. The networks of IRS1 and IRS2 in Huh7 and HCV-Huh7 cells were constructed. The networks indicate that in Huh7 cells, IRS1 is activated by Kruppel-like factor 10 (KLF10), IRS2, and four and a half LIM domains 2 (FHL2), and inhibited by solute carrier family 7 (cationic amino acid transporter, y + system), member 1 (SLC7A1), and IRS1 did not regulate any genes itself. IRS 2 is activated by thioredoxin interacting protein (TXNIP), KLF10, activating transcription factor 3 (ATF3) and IRS2, and inhibited by reticulocalbin 1 (RCN1), FHL2, suppressor of cytokine signaling 2 (SOCS2), stanniocalcin 2 (STC2), inhibin $\beta$ E (INHBE) and SLC7A1, while IRS2 activated oncostatin $\mathrm{M}$ receptor, TXNIP, RCN1, prion protein, B3 domain-containing proteinLOC_Os12g40080-like (LOC100128809), KLF10, ATF3, phosphoenolpyruvate carboxykinase 2, FHL2, SOCS2, STC2, interferon regulatory factor 9 (IRF9), asparagine synthetase (glutamine-hydrolyzing) (ASNS), brain-derived neurotrophic factor, chromosome 10 open reading frame 10 (C10orf10), IRS2, solute carrier family 1 (glutamate/neutral amino acid transporter), member 4 (SLC1A4), transforming growth factor $\beta 1$ induced transcript 1 (TGFB1I1), RAR-related orphan receptor A (RORA), SLC7A1, SLC1A4, LY6/PLAUR domain containing 1, LOC100134073, fatty acid binding protein 3, WD repeat domain 33, PPARG coactivator $1 \alpha$ (PPARGC1A), phospholipase A1 member A (PLA1A), Ras-related GTP binding D, basic helix-loop-helix family member e41 (BHLHE41), InaD-like (Drosophila), arrestin domain containing 4, zinc finger protein 295 (ZNF295), RORA, phospholipase D family member 6 (PLD6), 
Table I. Top 50 significant genes identified by significance analysis of microarray analysis.

\begin{tabular}{|c|c|c|}
\hline Gene ID & Gene name & Fold change \\
\hline 201010_s_at & Thioredoxin interacting protein & 21.518297 \\
\hline 203438_at & Stanniocalcin 2 & 20.873947 \\
\hline 210587_at & Inhibin, $\beta E$ & 19.26132 \\
\hline 201008_s_at & Thioredoxin interacting protein & 15.231962 \\
\hline 203439_s_at & Stanniocalcin 2 & 14.20261 \\
\hline 201009_s_at & Thioredoxin-interacting protein) & 13.7505045 \\
\hline 238029_s_at & Solute carrier family 16 (monocarboxylic acid transporters), member 14 & 11.429825 \\
\hline 205047_s_at & Asparagine synthetase (glutamine-hydrolyzing) & 11.315236 \\
\hline 231202_at & Aldehyde dehydrogenase 1 family, member L2 & 11.254294 \\
\hline 225283_at & Arrestin domain containing 4 & 10.549104 \\
\hline 228653_at & Sterile $\alpha$ motif domain containing 5 & 9.061874 \\
\hline 202672_s_at & Activating transcription factor 3 & 8.782885 \\
\hline 201300_s_at & Prion protein & 7.5039783 \\
\hline 212909_at & LY6/PLAUR domain containing 1 & 7.415823 \\
\hline 218332_at & Brain expressed gene 1 & 7.3808937 \\
\hline 203372_s_at & Suppressor of cytokine signaling 2 & 7.347132 \\
\hline 219195_at & Peroxisome proliferator-activated receptor $\gamma$, coactivator $1 \alpha$ & 6.9846096 \\
\hline 212810_s_at & Solute carrier family 1 (glutamate/neutral amino acid transporter), member 4 & 6.877748 \\
\hline 210426_x_at & RAR-related orphan receptor A & 6.6006436 \\
\hline 209183_s_at & Chromosome 10 open reading frame 10 & 6.530727 \\
\hline 226682_at & RAR-related orphan receptor $\alpha$ & 6.4361844 \\
\hline 202393_s_at & Kruppel-like factor 10 & 6.4241524 \\
\hline 214285_at & Fatty acid binding protein 3 , muscle and heart & 6.3771186 \\
\hline 203882_at & Interferon regulatory factor 9 & 6.129926 \\
\hline 221523_s_at & Ras-related GTP binding D & 6.011061 \\
\hline 212295_s_at & Solute carrier family 7 (cationic amino acid transporter, y + system), member 1 & 5.976633 \\
\hline 1554008_at & Oncostatin M receptor & 5.8743997 \\
\hline 218851_s_at & SFT2 domain containing 3 WD repeat domain 33 & 5.831587 \\
\hline 223681_s_at & InaD-like (Drosophila) & 5.778195 \\
\hline 203373_at & Suppressor of cytokine signaling 2 & 5.741121 \\
\hline 209651_at & Transforming growth factor $\beta 1$ induced transcript 1 & 5.6655536 \\
\hline 201063_at & Reticulocalbin 1 & 5.624495 \\
\hline 1569433_at & Sterile $\alpha$ motif domain containing 5 & 5.4978223 \\
\hline 206382_s_at & Brain-derived neurotrophic factor & 5.459223 \\
\hline 221530_s_at & Basic helix-loop-helix family, member e41 & 5.424661 \\
\hline 228708_at & RAB27B, member RAS oncogene family & 5.3274593 \\
\hline 209185_s_at & Insulin receptor substrate 2 & 5.273203 \\
\hline 209610_s_at & Solute carrier family 1 (glutamate/neutral amino acid transporter), member 4 & 5.230212 \\
\hline 214755_at & UDP-N-acteylglucosamine pyrophosphorylase 1-like 1 & 5.206161 \\
\hline 202847_at & Phosphoenolpyruvate carboxykinase 2 (mitochondrial) & 5.199079 \\
\hline 227037_at & Phospholipase D family, member 6) & 5.078215 \\
\hline 225539_at & Zinc finger protein 295 & 5.0584846 \\
\hline 210479_s_at & RAR-related orphan receptor $\alpha$ & 5.0370793 \\
\hline 212290_at & Solute carrier family 7 (cationic amino acid transporter, $y+$ system), member 1 & 5.0187483 \\
\hline 228519_x_at & Cold inducible RNA binding protein & 4.95052 \\
\hline 219584_at & Phospholipase A1 member A & 4.935018 \\
\hline 242979_at & Insulin receptor substrate 1 & 4.919549 \\
\hline 202949_s_at & Four and a half LIM domains 2 & 4.856401 \\
\hline 1568813_at & B3 domain-containing proteinLOC_Os12g40080-like & 4.792747 \\
\hline 212811_x_at & Solute carrier family 1 (glutamate/neutral amino acid transporter), member 4 & 4.7892227 \\
\hline
\end{tabular}


sterile $\alpha$ motif domain containing 5 (SAMD5), RAB27B member RAS oncogene family, solute carrier family 16 member 14 (SLC16A14) and IRS1, and inhibited SAMD5, INHBE and UDP-N-acetylglucosamine pyrophosphorylase 1 like 1. In HCV-Huh7 cells, IRS1 was activated by ATF3 and ASNS, and inhibited by INHBE and RCN1, and IRS1 did not regulate any genes. IRS2 was activated by ATF3, STC2, ASNS and SLC7A1, and inhibited by TXNIP, RCN1, IRS2 and INHBE. IRS2 activated SOCS2, C10orf10 and SLC16A14, however inhibited KLF10, IRS2, SLC7A1, PLA1A and ZNF295. The the network of IRS1 and IRS2 in Huh7 and HCV-Huh7 cells is presented in Fig. 1.

MAS 3.0 network analysis. The IRS1 and IRS2 sub-networks in Huh7 and HCV-Huh7 cells were then compared and the number of genes in each network was statistically analyzed by inputting the genetic codes into MAS 3.0 software, the enriched pathways and the GO networks. In Huh7 cells, 4 genes were upstream of IRS1, of which, 3 activate IRS1, and 1 inhibits IRS1; the former are enriched in 14 GOs and 3 pathways, while the latter is enriched in $3 \mathrm{GOs}$, however zero pathways. No gene was identified downstream of IRS1; therefore, none were enriched in GO and pathways. In HCV-Huh7 cells, 4 genes are upstream of IRS1, of which, 2 activate IRS1, and 2 inhibit IRS1; the former are enriched in 7 GOs and 2 pathways, while the latter are not enriched in any GOs or pathways. No gene was identified downstream of IRS1 and subsequently, none were enriched in any GO and pathways.

IRS2 sub-networks in Huh7 cells are more abundant when compared with IRS1 sub-networks. In Huh7 cells, 13 genes are upstream of IRS2. A total of 4 are functional enhancement genes and are enriched in 3 pathways and $16 \mathrm{GO}, 9$ are suppressor genes and are enriched in 5 pathways and 21 GOs. Notably, 45 genes are downstream of IRS2. Among them, 41 are activated by IRS2 and enriched in 15 pathways and 139 GOs and 3 are inhibited by IRS2 and enriched in 2 pathways and 4 GOs. In HCV-Huh7 cells, 8 genes are upstream of IRS2 and 8 genes are downstream of IRS2. Among the 8 upstream genes, 4 are functional enhancement genes and enriched in 2 pathways and 4 GOs; 4 are function suppressor genes and enriched in 5 pathways and 10 GOs. From the 8 downstream genes, 3 are activated by IRS2 and enriched in 3 pathways and 7 GOs and 5 are inhibited by IRS 2 and enriched in 4 pathways and 18 GOs. These results indicated that compared with IRS1, IRS2 is more active in Huh7 cells, particularly in its activated downstream genes and their corresponding pathways and GOs. However, following HCV infection, its activated downstream genes, their corresponding pathways and GOs reduced from 43, 15 and 139 to 3,3 and 7, respectively.

Network gene function module. To verify the biological information obtained during the MAS 3.0 analysis, the DAVID Gene Functional Classification Tool software was used to enrich the functional modules of the sub-networks of IRS1 and IRS2 in Huh7 and HCV-Huh7 cells. The enrichment results indicated that there are no activation and inhibition functional modules downstream and upstream of IRS1 in Huh7 and HCV-Huh7 cells, and no activation and inhibition modules downstream and upstream of IRS2 in Huh7 and HCV-Huh7 cells with the exception of 3 activation functional modules being enriched in the downstream of IRS2, cluster 1, cluster 2 and cluster 3 . The functions of cluster 1, include transcription factor, repressor, DNA-binding region, basic motif, identical protein binding, DNA binding, transcription regulation, transcription, transcription regulator activity, regulation of transcription, DNA-dependent, regulation of transcription, regulation of RNA metabolic process, transcription factor activity, nucleus and DNA binding. The functions of cluster 2, include activator, DNA binding, androgen signaling pathway, steroid hormone receptor binding, intracellular receptor-mediated signaling pathway, nuclear hormone receptor binding, intracellular signaling cascade, transcription cofactor activity, transcription coactivator activity, transcription factor binding, transcription activator activity, androgen receptor binding, hormone receptor binding, zinc-finger, regulation of transcription from RNA polymerase II promoter, positive regulation of macromolecule biosynthetic process, positive regulation of cellular biosynthetic process, positive regulation of macromolecule metabolic process, positive regulation of transcription DNA-dependent, positive regulation of DNA metabolic process, positive regulation of transcription, positive regulation of gene expression, positive regulation of nucleobase, nucleoside, nucleotide and nucleic acid metabolic process, positive regulation of regulation of nitrogen compound metabolic process, transcription regulation, transcription, phosphoprotein, zinc, transition metal ion binding, metal-binding, zinc ion binding, metal ion binding, cation binding, ion binding, transcription regulation activity, regulation of transcription, DNA-dependent, regulation of RNA metabolic, regulation of transcription. The functions of cluster 3, include glycoprotein, glycosylation site: $\mathrm{N}$-linker(GlcNAc), plasma membrane, transport, topological domain, extracellular, topological domain: Cytoplasmic, intrinsic to membrane, membrane, transmembrane region, transmembrane and integral to membrane.

Network gene signaling pathways. To understand the signaling pathways affecting the functions of IRS1 and IRS2 following $\mathrm{HCV}$ infection of Huh7 cells, the networking genes in Huh7 and HCV-huh7 cells were further analyzed using MAS 3.0 and enrichments of their index of pathways.

\section{Discussion}

The liver is important for the regulation of glucose metabolism due to the fact that it is a source of endogenous glucose and a vital organ for the insulin metabolism $(8,9)$. Chronic HCV infection is a multi-dysfunctional disease associated with insulin resistance, including associations with type 2 diabetes disorders (10-13). The pathophysiological and pathogenesis alterations that $\mathrm{HCV}$ infection triggers include insulin secretory defects, increased glycogen production and insulin resistance among others. Patients with insulin resistance require insulin to maintain their blood sugar at a normal level as they cannot maintain a steady glucose state without intervention $(14,15)$. In addition, 24-50\% patients with $\mathrm{HCV}$ have type 2 diabetes, and the incidence of $\mathrm{HCV}$-induced type 2 diabetes is 5 -fold greater than that induced by other causes of liver cirrhosis $(16,17)$. In addition, epidemiological studies have demonstrated that 
A
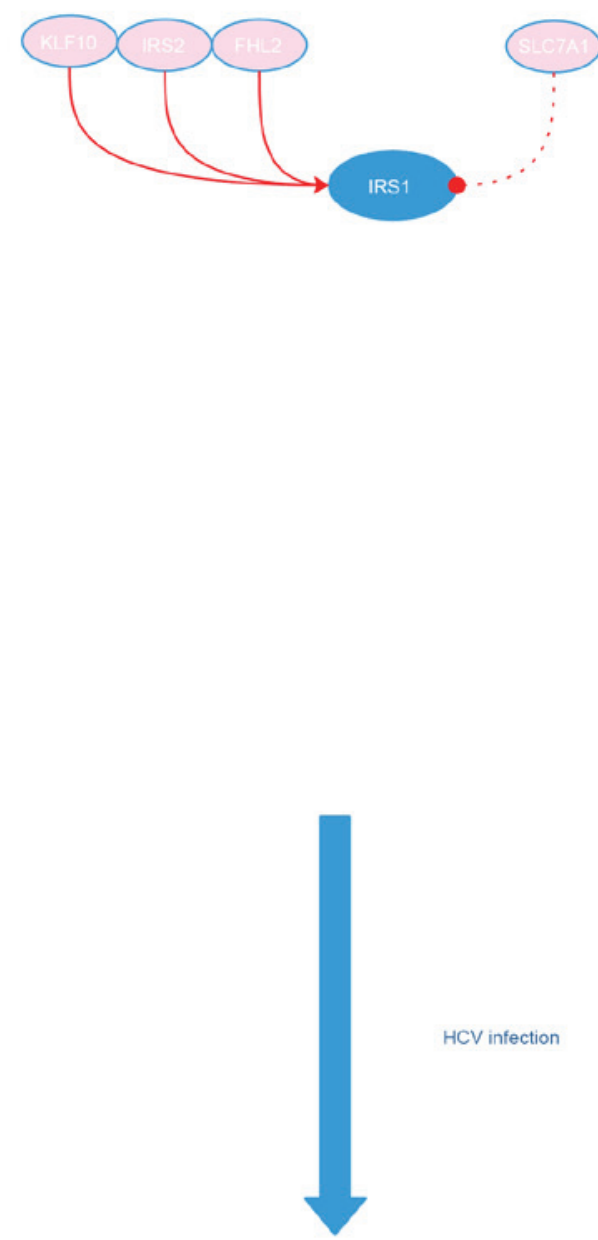

C

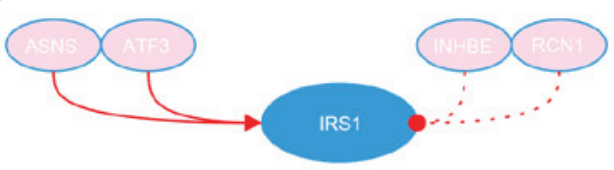

B

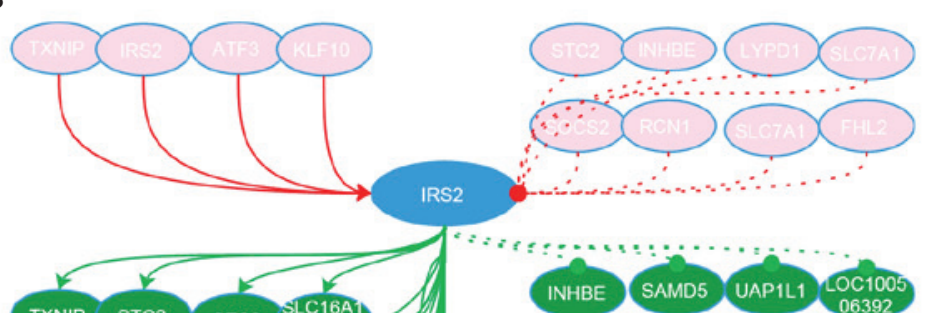

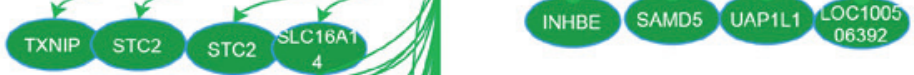

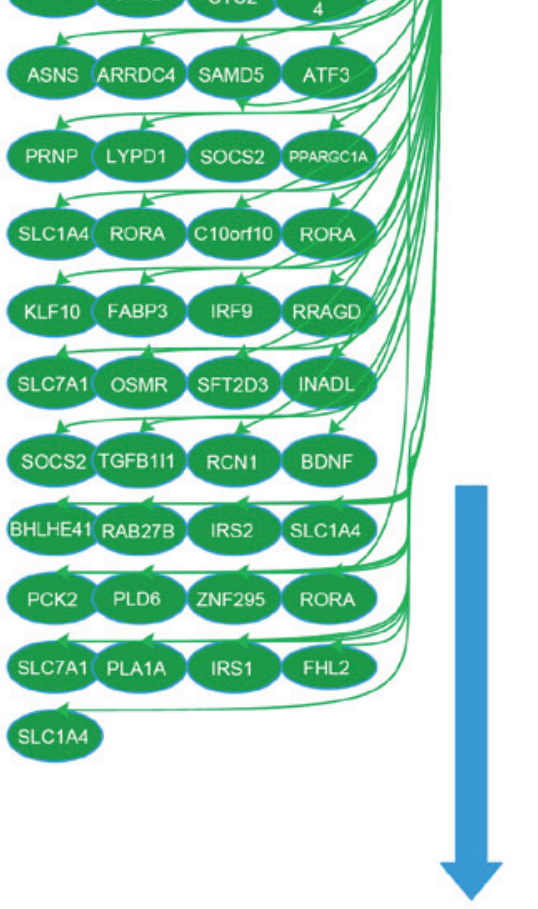

HCV infection

D

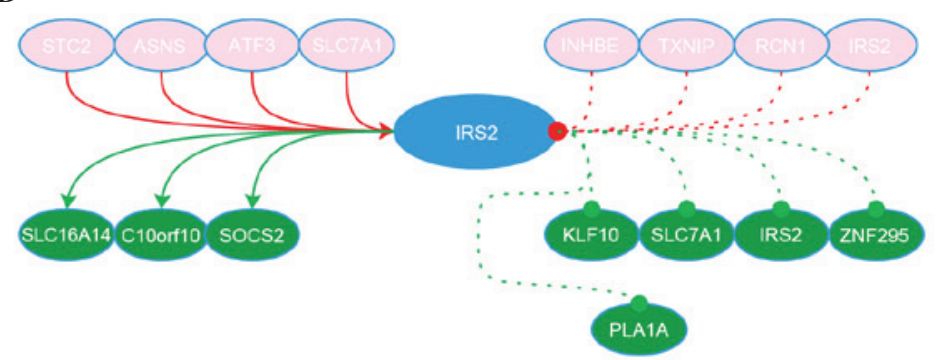

Figure 1. IRS1 and IRS2 network in Huh7 and HCV-infected Huh7 cells. (A) IRS1 network in Huh7 cells. (B) IRS2 network in Huh7 cells. (C) IRS1 network in HCV-Huh7 cell lines. (D) IRS2 network in HCV-Huh7 cell lines. Red circle with gene name indicates the genes upstream of IRS1 or IRS2. Green circle with gene name indicates the genes downstream of IRS1 or IRS2. Solid red line with red arrow indicates the activation of the gene in the upstream of IRS1 or IRS2 to IRS1 or IRS2. Dashed red line with red dot indicates the inactivation role of the gene upstream of IRS1 or IRS2 to IRS1 or IRS2. Solid green line with green arrow indicates the activation role of IRS1 or IRS2 to the gene downstream of IRS1 or IRS2. Dashed green line with green dot indicates the inhibitory role of IRS1 or IRS2 to the gene downstream of IRS1 or IRS2. IRS, insulin receptor substrate; HCV, hepatitis C virus; KLF10, kruppel-like factor 10; STC2, stanniocalcin 2; INHBE, inhibin $\beta E$; LYPD1, LY6/PLAUR domain containing 1; SOCS2, suppressor of cytokine signaling 2; RCN1, EF-hand calcium binding domain; FHL2, four and a half LIM domains 2; SLC16A14, solute carrier family 16 (monocarboxylic acid transporters), member 14; ASNS, asparagine synthetase (glutamine-hydrolyzing); ARRDC4, arrestin domain containing 4; SAMD5, sterile $\alpha$ motif domain containing 5; ATF3, activating transcription factor 3; PRNP, prion protein; PPARGC1A, peroxisome proliferator-activated receptor $\gamma$, coactivator 1 $\alpha$; OSMR, oncostatin M receptor; SFT2D3 WDR33, SFT2 domain containing3 WD repeat domain 33; INADL, InaD-like (Drosophila); TGFB1I1, transforming growth factor $\beta 1$ induced transcript 1; BDNF, brain-derived neurotrophic factor; BHLHE41, basic helix-loop-helix family, member e41; RAB27B, RAB27B member RAS oncogene family; PCK2, phosphoenolpyruvate carboxykinase 2 (mitochondrial); PLD6, phospholipase D family, member 6; ZNF295, zinc finger protein 295; PLA1A, phospholipase A1 member A; SAMD5, sterile $\alpha$ motif domain containing 5; UAP1L1, UDP-N-acteylglucosamine pyrophosphorylase 1-like 1; LOC100506392, B3 domain-containing proteinLOC_Os12g40080-like.

cirrhotic patients with $\mathrm{HCV}$ infection are correlated with cases of type 2 diabetes (18-26). However, novel evidence has indicated that the incidence of type 2 diabetes in non-cirrhotic
$\mathrm{HCV}$-infected patients is higher than that in age-matched, non-cirrhotic hepatitis B virus-infected patients or that of healthy controls (27), suggesting that HCV-induced insulin 
resistance may begin at the non-fibrosis stage of $\mathrm{HCV}$-infected patients.

The exact pathogenesis of $\mathrm{HCV}$-induced insulin resistance remains unclear, however a number of hypotheses have been suggested. Previous studies have indicated that HCV may increase the expression of proinflammatory cytokine tumor necrosis factor $\alpha(\mathrm{TNF} \alpha)$ and interleukin 6 (IL-6) (28), interfere with IRS1 tyrosine phosphorylation, enhance the damage of insulin signaling pathways and promote insulin resistance $(29,30)$. In addition, $\mathrm{TNF} \alpha$ has a direct toxic effect on pancreatic $\beta$ cells in vitro, and may reduce insulin secretion, leading to insulin resistance (31). Additionally, HCV core gene transgenic mice are insulin-resistant and exhibit with hepatic insulin dysfunction (29).

HCV core proteins may stimulate cytokine signal transduction and enhance the ubiquitination of the IRS1 and IRS2 proteasomes, thus resulting in functional degradation $(32,33)$. The HCV non-structural protein 5A may increase the expression of protein phosphatase 2A (PP2A), which dephosphorylates protein kinase $\mathrm{B}(\mathrm{Akt})$ and subsequently reduces Akt activity (34). Defection of insulin signaling due to IRS1 tyrosine kinase phosphorylation and phosphoinositide 3-kinase (PI3K) activation may promote insulin resistance $(32,35,36)$, leading to type 2 diabetes.

Therefore, the current study aimed to use bioinformatics in order to deduce the possible roles of IRS1 and IRS2 in Huh7 and HCV-Huh7 cells. In a previous study, the construction and analysis of gene networks associated with ATF3 were investigated (37). The results of the present study indicated that no gene is downstream of IRS1 in Huh7 and HCV-Huh7 cells, and no corresponding GO or pathway is enriched. However, 43 genes are downstream of IRS2, and these genes are enriched in 139 GOs, 15 pathway, and 3 clusters in Huh7 cells. Following HCV infection, only 3 genes are downstream of IRS2, and are enriched in 7 GOs and 3 pathways, indicating that the IRS2 downstream function is significantly redcued by HCV infection. When comparing the downstream modules of IRS1 with the IRS2 sub-networks in Huh7 cells, the number of genes, pathways, GO and functional modules downstream of IRS1 is zero. IRS1 function is silenced, however IRS2 function is active.

Previous studies have indicated that insulin may regulate glucose concentration and increase glycogen synthesis in the liver by preventing gluconeogenesis and glycogen decomposition $(38,39)$. It performs these functions through IRS1 and IRS2, which belong to the cytoplasmic adapter proteins primarily connecting the IR and various of effector molecules, leading to a cellular response to insulin. IRS2 is the major protein of the IRS family distributed in the liver. Upon the binding of insulin to the IR, the tyrosine residues in the proximal membrane region of the $\beta$ subunit of IR undergoes autophosphorylation and binds to IRS2. The activated tyrosine kinase of the insulin receptor (protein-tyrosine kinase, insulin receptor tyrosine kinase) further phosphorylates IRS2 at multiple tyrosine residues, providing binding sites for Src homology 2 domains of downstream proteins. The formed signaling protein complexes further mediate signal transduction, thereby regulating glucose metabolism, gene expression and cell division and controlling cell growth, differentiation and metabolism (40). These studies are in agreementwith the observations presented in the current study. From the constructed downstream molecular network of IRS1 and IRS2 (Fig. 1), it is suggested that in Huh7 cells, only 4 genes are upstream of IRS1, 3 of which are functional enhancement genes and are enriched in 14 GOs and 3 pathways, and 1 is a suppressing gene enriched in 3 GOs, however zero pathways and no genes were identified downstream of IRS1 in Huh7 cells. In HCV-Huh7 cells, 4 genes are upstream of IRS1, 2 of which are functional enhancement genes and enriched in 7 GOs and 2 pathways, 2 of which are suppressing genes and enriched in zero GOs and pathways, with no genes downstream of IRS1. Compared with IRS1, IRS2 exhibited more abundant gene networks in Huh7 cells. There are 13 genes upstream of IRS2, 4 of which are functional enhancement genes and enriched in 3 GOs and 16 pathways, and 9 that are suppressing genes and enriched in 5 GOs and 21 pathways. Notably, there are 45 genes downstream of IRS2 in Huh7 cells, of which, 41 are functional enhancement genes and enriched in 15 GOs and 139 pathways, and 3 are suppressing genes and enriched in 2 GOs and 4 pathways. In HCV-Huh7 cells, there are 8 genes upstream of IRS2 and 8 genes downstream of IRS2. In the upstream genes, 4 are functional enhancement genes and enriched in $2 \mathrm{GO}$ and 7 pathways, and 4 are suppressing genes and enriched in 5 GOs and 10 pathways. In the downstream genes, 3 are functional enhancement genes and enriched in 3 GOs and 7 pathways, and 5 are suppressing genes and enriched in 4 GOs and 18 pathways. Therefore, IRS1 is suggested to be functionally quiescent in Huh7 and HCV-Huh7 cells and IRS2 was identified as more active in Huh7 cells with richer gene networks, in particular in its functional enhancement genes. However, in HCV-Huh7 cells, the number of network genes was significantly reduced, although remained at a low level, suggesting that following $\mathrm{HCV}$ infection, IRS may preserve certain IRS2-mediated functions. Therefore, it is possible that IRS2 is the major functional IRS in Huh7 cells and HCV infection greatly diminishes its functions to a very low level, which is consistent with previous studies. Previous studies have indicated that knockout IRS2 in normally developed mice may induce diabetes, including insulin resistance (41), while a knockout of IRS1 induces growth retardation and insulin resistance, however not diabetes $(42,43)$. However, knockout of IRS1 and IRS2 may result in hyperinsulinemia and insulin resistance in the liver (44). Previous reports on patients with insulin resistance and hyperglycemia (45) and obese/diabetic mice (46) determined that their IRS1 and IRS2 levels were reduced. The expression of the HCV core protein may reduce tyrosine phosphorylation of IRS1 and reduce IRS2 expression in the liver; however, does not completely eradicate their activity, allowing the remaining IRS to transduct weak signals to their downstream molecules (47).

The constructed networks indicate that HCV infection may lead to downstream dysfunction of IRS in Huh7 cells and aberrant insulin metabolism. Modulation of IRS1 and IRS2 in Huh7 and HCV-Huh7 cells, which were enriched using the DAVID gene functional classification tool, indicated that there are only 3 enriched functional modules downstream of IRS2 in Huh7 cells, and no other functional modules for IRS2 and IRS1. Previous studies on IRS2 have primarily focused on glucose metabolism (48-50). The present study indicated 
that downstream signals of IRS2 are involved in not only glucose metabolism, but additionally in DNA transcription, metal-binding regulation, membrane function, hormonal regulation function, signaling transduction, macromolecular biosynthesis and regulation, signaling pathways regulating function and extracellular and cytoplasmic domain topology structure function. The IRS2 downstream modules are fully blocked by HCV infection, which can directly interfere with insulin signaling transduction, thereby leading to insulin resistance. The genes associated with these functions include IRF9, BHLHE41, ATF3, KLF10, RORA, TGFB1I1, ZNF295, PPARGC1A, FHL2, SLC16A14, SLC16A14, PLD6 and SLC7A1 and should be considered in future studies.

Studies investigating $\mathrm{HCV}$-induced insulin signaling disorders are rare. It has been determined that the peroxisome proliferator-activated receptors (PPARs) are associated with nuclear factors, and in particular are capable of regulating glucose homeostasis. In addition, the association between HCV replication and expression of PPARs has drawn attention from researchers. PPARs are ligand-activated transcription factors belonging to the nuclear receptor superfamily (51) and require retinoic acid to maintain receptor heterologous dimerization $(52,53)$. The PPAR family includes PPAR $\alpha$, PPAR $\beta$, PPAR $\delta$ and PPAR $\gamma$ (51). These PPARs regulate cell differentiation, growth and metabolism. PPAR $\alpha / \gamma$ along with their exclusive partner the retinoid $\mathrm{X}$ receptor are nuclear receptors expressed predominantly in the liver (54). PPAR $\alpha$ may upregulate glycerol phosphate dehydrogenase, glycerol kinase and glycerol transport proteins, all of which may synthesize glucose in a fasting state (55). PPAR agonists activate insulin signaling pathways in living tissues in a time-dependent manner (56). Biopsy of patients with chronic $\mathrm{HCV}$ indicated reduced PPAR- $\alpha$ mRNA levels $(53,57)$. A previous in vitro study determined that reduced expression of PPAR $\gamma$ in Huh7 cells infected with HCV core protein 3 (58), suggesting that PPARs are involved in $\mathrm{HCV}$-induced insulin resistance.

HCV may also activate endoplasmic reticulum (ER) stress (59). Increased ER stress may inhibit insulin signaling by phosphorylating the c-Jun N-terminal kinase (JNK) family and IRS1 (60). In addition, ER stress may activate PP2A (61) and thus inhibit Akt kinase and adenosine monophosphate kinase and regulate gluconeogenesis (59).

$\mathrm{HCV}$ infection may activate extracellular signal-regulated kinases $1 / 2$ by its forkhead box O1 (FOXO1) site, S253, through activation of p38 mitogen-activated protein kinase (MAPK) phosphatase-3 (MKP-3) and PP2A, and further promote gluconeogenesis. Interaction of MKP3 or PP2A with FOXO1 may lead to dephosphorylation of FOXOl at the S253 site and its activation $(62,63)$. FOXOl mutations may result in developments of metabolism disorders and organ failure. FOXOl promotes glycogen production and inhibits lipogenesis by upregulating phosphoenolpyruvate carboxykinase and glucose-6-phosphatase, catalytic subunit expression (64-66). Upon $\mathrm{HCV}$ infection, its core protein induces production of SOCS-3 and promotes degradation of IRS1 and IRS2 through ubiquitination and degradation of the proteasome. Downregulation of IRS1 and IRS2 inhibits insulin signal transduction, leading to insulin resistance $(32,35,36)$. A recent study identified that $\mathrm{HCV}$ infection may lower the expression of phosphatase and tensin homolog deleted on chromosome 10 (PTEN), which in turn increases phosphorylation of IRS1 at Ser307 and inhibits the PI3K/Akt signaling pathway, leading to insulin resistance (67).

In summary, previous studies have determined that PP2A, JNK, SOCS-3, PTEN and other signaling pathways are involved in $\mathrm{HCV}$-induced hepatic insulin resistance, including PP2A and SOCS-3, which is consistent with the results of the current study deduced from the network information. Fig. 2 compares the pathogenic mechanisms underlying $\mathrm{HCV}$ infection-induced insulin resistance identified in the present study with previous literature.

Aytug et al (34) first reported that HCV infection may impede the IRS1/PI3K signal pathway. However, the present study indicated that IRS2 signaling is the major pathway responsible for $\mathrm{HCV}$ infection-induced insulin resistance in Huh7 cells. The enrichment results of the current study indicated that following $\mathrm{HCV}$ infection of Huh7 cells, IRS2-activated signaling pathways are reduced in terms of quantity and type, suggesting that IRS2 is important in hepatic insulin resistance. In contrast, no alterations in the IRS1 signaling pathway were observed, which is not consistent with the results of Aytug et al (34). Previous studies have indicated that chronic HCV infection-induced reduction of the responsiveness of tissues to insulin and insulin resistance may be associated with reduced IRS2 expression (32,68-71).

The results of the current study indicated that $\mathrm{HCV}$ infection-induced insulin resistance in Huh7 cells is not completely due to reduced expression of IRS1 or IRS2. $\mathrm{HCV}$ infection-induced blockage of IRS2 downstream pathways may also be an important factor. This blockage at non-fibrous HCV infection period does not lead to reduced IRS1 and IRS2 expression. In contrast, HCV infection may increase IRS1 and IRS2 expression in Huh7 cells; however, the downstream activated signaling pathways of IRS2 were severely disrupted in the current study. It is suggested that the former is a feedback of the latter. HCV-induced low insulin responsiveness and insulin resistance are potentially due to $\mathrm{HCV}$-induced reduced expression of IRS1 and IRS2, in addition to $\mathrm{HCV}$-induced direct blockage of downstream signaling of IRS2. Clinical studies have demonstrated that serum IRS1 levels in 42 non-diabetic, $\mathrm{HCV}$-infected patients were 2 -fold of that of non-HCV infected controls (34).

Previous studies on IRS2 have been predominantly focused upon the glucose metabolism. DAVID functional modeling in Huh7 cells determined 3 enriched functional modules downstream of IRS2 activation, indicating that IRS2 may be widely involved in transcriptional regulation, zinc finger transcription regulation, nuclear regulation, membrane function, hormonal regulation in Huh7 cells. In addition, HCV infection fully blocks these functions of IRS2, which may directly interfere with insulin signal transduction and lead to insulin resistance. In addition to cellular network modules, IRS1 and IRS2 signaling pathways were identified in Huh7 cells. No signaling pathways are activated by IRS1, while multiple pathways are activated by IRS2, including insulin signaling pathway, adipocytokine signaling pathway, type 2 diabetes mellitus, Jak-signal transducer and activator of transcription (STAT) signaling pathway, PPAR signaling pathway, alkaloid biosynthese II, circadian rhythm, nitrogen metabolism, alanine and aspartate 


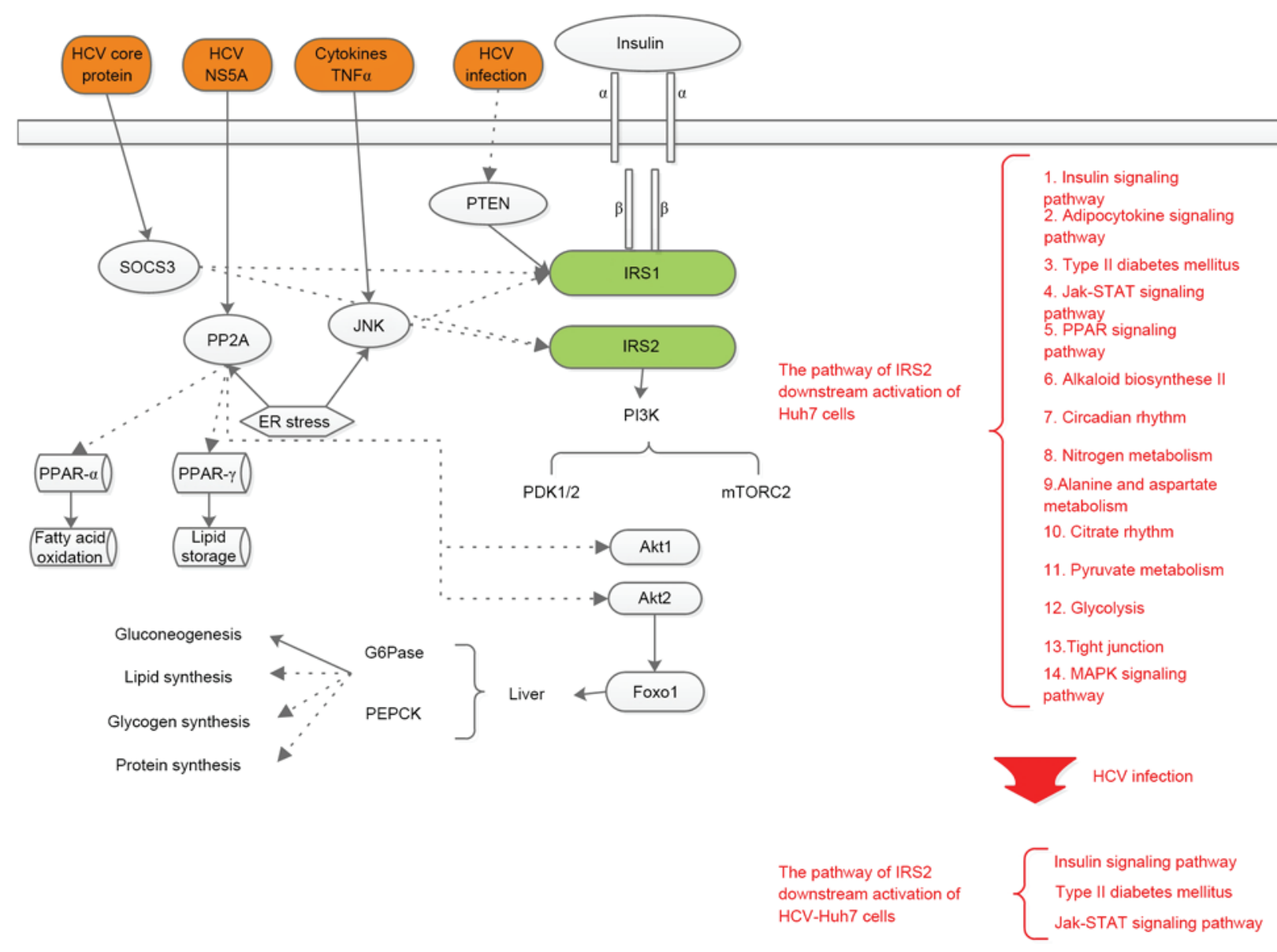

Figure 2. Comparison with the hypothesis of previous literature and the present study. Solid lines with an arrow indicate strengthening effects, dashed lines with an arrow indicate inhibitory effects. Black text indicates the hypothesis of previous literature, red text indicates the hypothesis of the current study. $\mathrm{HCV}$, hepatitis $\mathrm{C}$ virus; NS5A, non-structural protein $5 \mathrm{~A}$; TNF $\alpha$, tumor necrosis factor $\alpha$; PP2A, protein phosphatase 2A; JNK, c-Jun N-terminal kinase; ER, endoplasmic reticulum; IRS1/2, insulin receptor substrate 1/2; PI3K, phosphoinositide 3-kinase; PDK1, 3-phosphoinositide-dependent kinase 1; mTOR, mammalian target of rapamycin; Akt, protein kinase B; MAPK, p38 mitogen-activated protein kinase; FOXO1, forkhead box O1; G6Pase- $\alpha$, glucose-6-phosphatase- $\alpha$; PEPCK, phosphoenolpyruvate carboxykinase; JAK, janus kinase; STAT, signal transducer and activator of transcription; PTEN, phosphatase and tensin homolog deleted on chromosome 10.

metabolism, citrate rhythm, pyruvate metabolism, glycolysis, tight junction, cytokine-cytokine receptor interaction and the MAPK signaling pathway. Their corresponding enrichment numbers are 5, 4, 3, 3, 2, 1, 1, 1, 1,1,1,1,1,1 and 1, respectively. Following HCV infection, no signaling pathway was activated by IRS1, and only 3 signaling pathways were activated by IRS2, including the insulin signaling pathway, type 2 diabetes mellitus and Jak-STAT signaling pathway. Their corresponding enrichment numbers were reduced to 1,1 and 1, respectively. It is possible that HCV infection-induced blockage of insulin signaling in Huh7 cells is mediated by IRS 2 because following $\mathrm{HCV}$ infection, the number and type of signaling pathways activated by IRS 2 were significantly reduced while those of IRS1 underwent no changes. Thus, IRS2 may be important in hepatic insulin resistance.

In conclusion, by comparing the gene networks activated/inhibited by IRS1 and IRS2 in Huh7/HCV-Huh7 cells, the present study determined that HCV may impact IRS1 and IRS2 signaling pathways in Huh7 cells, leading to blockage of IRS pathways and thereby liver-derived insulin resistance. Such a signaling blockage primarily affects downstream pathways of IRS2. In addition, this blockage affects multiple aspects, including metabolism, transcription regulation, zinc finger transcription regulation, nuclear regulation, membrane function and hormone regulation. Furthermore, in addition to the reported blockage of the PPAR signaling pathway and cytokine-cytokine receptor interaction, the bioinformatic analysis of the present study indicated that HCV infection may also disturb multiple pathways downstream of IRS2, which may lead to HCV-induced hepatic insulin resistance. The following signaling pathways should also be investigated: Insulin signaling pathway, adipocytokine signaling pathway, type 2 diabetes mellitus, Jak-STAT signaling pathway, PPAR signaling pathway, alkaloid biosynthese II, circadian rhythm, nitrogen metabolism, alanine and aspartate metabolism, citrate rhythm, pyruvate metabolism, glycolysis, tight junction including the MAPK signaling pathway, and the following genes involved in hepatic insulin resistance: IRF9, BHLHE41, ATF3, KLF10, RORA, TGFB1I1, ZnF295, PPARGC1A, FHL2, SLC16A14, PLD6 and SLC7A1. Overall, the present study constructed and analyzed IRS1 and IRS2 sub-networks in Huh7 and HCV-Huh7 cells aiming to gain insight into the cellular mechanisms underlying HCV-induced insulin resistance. 


\section{References}

1. Serfaty L and Capeau J: Hepatitis C, insulin resistance and diabetes: Clinical and pathogenic data. Liver Int 29 (Suppl 2): $13-25,2009$

2. Koike $\mathrm{K}$ and Moriya $\mathrm{K}$ : Metabolic aspects of hepatitis $\mathrm{C}$ viral infection: Steatohepatitis resembling but distinct from NASH. J Gastroenterol 40: 329-336, 2005.

3. Jian Wu Y, Shu Chen L and Gui Qiang W: Effects of fatty liver and related factors on the efficacy of combination antiviral therapy in patients with chronic hepatitis C. Liver Int 26 . 166-172, 2006

4. Xiong J, Lu Y, Feng J, Yuan D, Tian M, Chang Y, Fu C, Wang G, Zeng $\mathrm{H}$ and Miao W: Tetrahymena functional genomics database (TetraFGD): An integrated resource for Tetrahymena functional genomics. Database (Oxford) 12: bat008, 2013.

5. Wang Y, Joshi T, Zhang XS, Xu D and Chen L: Inferring gene regulatory networks from multiple microarray datasets. Bioinformatics 22: 2413-2420, 2006.

6. Huang DW, Sherman BT, Tan Q, Collins JR, Alvord WG, Roayaei J, Stephens R, Baseler MW, Lane HC and Lempicki RA: The DAVID Gene Functional Classification Tool: A novel biological module-centric algorithm to functionally analyze large gene lists. Genome Biol 8: R183, 2007.

7. Dennis G, Jr., Sherman BT, Hosack DA, Yang J, Gao W, Lane HC and Lempicki RA: DAVID: Database for Annotation, Visualization, and Integrated Discovery. Genome Biol 4: 3, 2003.

8. Kadowaki T: Insights into insulin resistance and type 2 diabetes from knockout mouse models. J Clin Invest 106: 459-465, 2000.

9. Ueki K, Yamauchi T, Tamemoto H, Tobe K, Yamamoto-Honda R Kaburagi Y, Akanuma Y, Yazaki Y, Aizawa S, Nagai R and Kadowaki T: Restored insulin-sensitivity in IRS-1-deficient mice treated by adenovirus-mediated gene therapy. J Clin Invest 105 : $1437-1445,2000$

10. Micallef JM, Kaldor JM and Dore GJ: Spontaneous viral clearance following acute hepatitis $\mathrm{C}$ infection: A systematic review of longitudinal studies. J Viral Hepat 13: 34-41, 2006.

11. Allison ME, Wreghitt T, Palmer CR and Alexander GJ Evidence for a link between hepatitis $\mathrm{C}$ virus infection and diabetes mellitus in a cirrhotic population. J Hepatol 21: 1135-1139, 1994

12. Cavaghan MK, Ehrmann DA and Polonsky KS: Interactions between insulin resistance and insulin secretion in the development of glucose intolerance. J Clin Invest 106: 329-333.

13. Kahn BB: Type 2 diabetes: When insulin secretion fails to compensate for insulin resistance. Cell 92: 593-596, 1998

14. Campbell PJ, Mandarino LJ and Gerich JE: Quantification of the relative impairment in actions of insulin on hepatic glucose production and peripheral glucose uptake in non-insulin-dependent diabetes mellitus. Metabolism 37: 15-21, 1988.

15. Romero-Gómez M, Del Mar Viloria M, Andrade RJ, Salmerón J, Diago M, Fernández-Rodríguez CM, Corpas R, Cruz M Grande L, Vázquez L, et al: Insulin resistance impairs sustained response rate to peginterferon plus ribavirin in chronic hepatitis $\mathrm{C}$ patients. Gastroenterology 128: 636-641, 2005.

16. Romero-Gómez M: Insulin resistance and hepatitis C. World J Gastroenterol 12: 7075-7080, 2006.

17. Arao M, Murase K, Kusakabe A, Yoshioka K, Fukuzawa Y, Ishikawa $\mathrm{T}$, Tagaya $\mathrm{T}$, Yamanouchi $\mathrm{K}$, Ichimiya $\mathrm{H}$, Sameshima Y and Kakumu S: Prevalence of diabetes mellitus in Japanese patients infected chronically with hepatitis $\mathrm{C}$ virus. J Gastroenterol 38: 355-360, 2003.

18. Mason AL, Lau JY, Hoang N, Qian K, Alexander GJ, Xu L, Guo L, Jacob S, Regenstein FG, Zimmerman R, et al: Association of diabetes mellitus and chronic hepatitis $\mathrm{C}$ virus infection. Hepatology 29: 328-333, 1999.

19. Fraser GM, Harman I, Meller N, Niv Y and Porath A: Diabetes mellitus is associated with chronic hepatitis $\mathrm{C}$ but not chronic hepatitis B infection. Isr J Med Sci 32: 526-530, 1996.

20. Caronia S, Taylor K, Pagliaro L, Carr C, Palazzo U, Petrik J, O'Rahilly S, Shore S, Tom BD and Alexander GJ: Further evidence for an association between non-insulin-dependent diabetes mellitus and chronic hepatitis $\mathrm{C}$ virus infection. Hepatology 30: 1059-1063, 1999.

21. Mehta SH, Brancati FL, Sulkowski MS, Strathdee SA, Szklo M and Thomas DL: Prevalence of type 2 diabetes mellitus among persons with hepatitis $C$ virus infection in the United States. Ann Intern Med 133: 592-599, 2000.
22. Grimbert S, Valensi P, Lévy-Marchal C, Perret G, Richardet JP, Raffoux C, Trinchet JC and Beaugrand M: High prevalence of diabetes mellitus in patients with chronic hepatitis C. A case-control study. Gastroenterol Clin Biol 20: 544-548, 1996.

23. Ozyilkan E, Erbaş T, Simşek H, Telatar F, Kayhan B and Telatar H: Increased prevalence of hepatitis $\mathrm{C}$ virus antibodies in patients with diabetes mellitus. J Intern Med 235: 283-284, 1994.

24. Simó R, Hernández C, Genescà J, Jardí R and Mesa J: High prevalence of hepatitis $\mathrm{C}$ virus infection in diabetic patients. Diabetes Care 19: 998-1000, 1996.

25. Zein NN, Abdulkarim AS, Wiesner RH, Egan KS and Persing DH: Prevalence of diabetes mellitus in patients with end-stage liver cirrhosis due to hepatitis $\mathrm{C}$, alcohol, or cholestatic disease. J Hepatol 32: 209-217, 2000

26. Hui JM, Sud A, Farrell GC, Bandara P, Byth K, Kench JG, McCaughan GW and George J: Insulin resistance is associated with chronic hepatitis $\mathrm{C}$ virus infection and fibrosis progression [corrected]. Gastroenterology 125: 1695-1704, 2003.

27. Alexander GJ: An association between hepatitis $\mathrm{C}$ virus infection and type 2 diabetes mellitus: What is the connection? Ann Intern Med 133: 650-652, 2000.

28. Oliveira CP, Kappel CR, Siqueira ER, Lima VM, Stefano JT, Michalczuk MT, Marini SS, Barbeiro HV, Soriano FG, Carrilho FJ et al: Effects of hepatitis $\mathrm{C}$ virus on cardiovascular risk in infected patients: A comparative study. Int J Cardiol 164: 221-226, 2013.

29. Shintani Y, Fujie H, Miyoshi H, Tsutsumi T, Tsukamoto K, Kimura S, Moriya K and Koike K: Hepatitis C virus infection and diabetes: Direct involvement of the virus in the development of insulin resistance. Gastroenterology 126: 840-848, 2004.

30. Zekri AR, Ashour MS, Hassan A, Alam El-Din HM, El-Shehaby AM and Abu-Shady MA: Cytokine profile in Egyptian hepatitis $\mathrm{C}$ virus genotype-4 in relation to liver disease progression. World J Gastroenterol 11: 6624-6630, 2005.

31. Greenberg AS and McDaniel ML: Identifying the links between obesity, insulin resistance and beta-cell function: Potential role of adipocyte-derived cytokines in the pathogenesis of type 2 diabetes. Eur J Clin Invest 32 (Suppl 3): 24-34, 2002

32. Kawaguchi T, Yoshida T, Harada M, Hisamoto T, Nagao Y, Ide T, Taniguchi E, Kumemura H, Hanada S, Maeyama M, et al: Hepatitis C virus down-regulates insulin receptor substrates 1 and 2 through up-regulation of suppressor of cytokine signaling 3 . Am J Pathol 165: 1499-1508, 2004.

33. Kawaguchi T, Ide T, Taniguchi E, Hirano E, Itou M, Sumie $\mathrm{S}$, Nagao Y, Yanagimoto C, Hanada S and Koga H: Clearance of HCV improves insulin resistance, beta-cell function, and hepatic expression of insulin receptor substrate 1 and 2 . Am J Gastroenterol 102: 570-576, 2007.

34. Aytug S, Reich D, Sapiro LE, Bernstein D and Begum N: Impaired IRS-1/PI3-kinase signaling in patients with HCV: A mechanism for increased prevalence of type 2 diabetes. Hepatology 38: 1384-1392, 2003

35. Banerjee S, Saito K, Ait-Goughoulte M, Meyer K, Ray RB and Ray R: Hepatitis $\mathrm{C}$ virus core protein upregulates serine phosphorylation of insulin receptor substrate- 1 and impairs the downstream akt/protein kinase B signaling pathway for insulin resistance. J Virol 82: 2606-2612, 2008.

36. Lecube A, Hernández C, Genescà J and Simó R: Proinflammatory cytokines, insulin resistance, and insulin secretion in chronic hepatitis C patients: A case-control study. Diabetes Care 29: 1096-1101, 2006

37. Liu J, Wang B, Wang W, Sun M, Li Y, Jia X, Zhai S and Dang S: Computational networks of activating transcription 3 gene in Huh7 cell lines and hepatitis C virus-infected Huh7 cell lines. Mol Med Rep 12: 1239-1246, 2015.

38. Brüning JC, Michael MD, Winnay JN, Hayashi T, Hörsch D, Accili D, Goodyear LJ and Kahn CR: A muscle-specific insulin receptor knockout exhibits features of the metabolic syndrome of NIDDM without altering glucose tolerance. Mol Cell 2: 559-569, 1998.

39. Kulkarni RN, Brüning JC, Winnay JN, Postic C, Magnuson MA and Kahn CR: Tissue-specific knockout of the insulin receptor in pancreatic beta cells creates an insulin secretory defect similar to that in type 2 diabetes. Cell 96: 329-339, 1999.

40. Vassen L, Wegrzyn W and Klein-Hitpass L: Human insulin receptor substrate-2: Gene organization and promoter characterization. Diabetes 48: 1877-1880, 1999.

41. Withers DJ, Gutierrez JS, Towery H, Burks DJ, Ren JM, Previs S, Zhang Y, Bernal D, Pons S, Shulman GI, et al: Disruption of IRS-2 causes type 2 diabetes in mice. Nature 391: 900-904, 1998. 
42. Araki E, Lipes MA, Patti ME, Brüning JC, Haag B III, Johnson RS and Kahn CR: Alternative pathway of insulin signalling in mice with targeted disruption of the IRS-1 gene. Nature 372: 186-190, 1994

43. Tamemoto H, Kadowaki T, Tobe K, Yagi T, Sakura H, Hayakawa T, Terauchi Y, Ueki K, Kaburagi Y, Satoh S, et al: Insulin resistance and growth retardation in mice lacking insulin receptor substrate-1. Nature 372: 182-186, 1994.

44. Taniguchi CM, Ueki K and Kahn R: Complementary roles of IRS-1 and IRS-2 in the hepatic regulation of metabolism. J Clin Invest 115: 718-727, 2005.

45. Anai M, Funaki M, Ogihara T, Terasaki J, Inukai K, Katagiri H, Fukushima Y, Yazaki Y, Kikuchi M, Oka Y and Asano T: Altered expression levels and impaired steps in the pathway to phosphatidylinositol 3-kinase activation via insulin receptor substrates 1 and 2 in Zucker fatty rats. Diabetes 47: 13-23, 1998.

46. Kerouz NJ, Hörsch D, Pons S and Kahn CR: Differential regulation of insulin receptor substrates-1 and -2 (IRS-1 and IRS-2) and phosphatidylinositol 3-kinase isoforms in liver and muscle of the obese diabetic (ob/ob) mouse. J Clin Invest 100: 3164-3172, 1997.

47. Miyamoto H, Moriishi K, Moriya K, Murata S, Tanaka K, Suzuki T, Miyamura T, Koike K and Matsuura Y: Involvement of the PA28gamma-dependent pathway in insulin resistance induced by hepatitis C virus core protein. J Virol 81: 1727-1735, 2007.

48. Tonelli J, Li W, Kishore P, Pajvani UB, Kwon E, Weaver C, Scherer PE and Hawkins M: Mechanisms of early insulin-sensitizing effects of thiazolidinediones in type 2 diabetes. Diabetes. 53: 1621-1629, 2004.

49. Wu HS, Yu JH, Li YY, Yang YS, He QJ, Lou YJ and Ji RY: Insulin-sensitizing effects of a novel alpha-methyl- alpha -phenoxylpropionate derivative in vitro. Acta Pharmacol Sin 28 : 417-422, 2007

50. Mousavinasab F, Tähtinen T, Jokelainen J, Koskela P, Vanhala M, Oikarinen J, Keinänen-Kiukaanniemi S and Laakso M: Effect of the Pro12Ala polymorphism of the PPARg2 gene on serum adiponectin changes: Endocrine 27: 307-309, 2005.

51. Yessoufou A and Wahli W: Multifaceted roles of peroxisome proliferator-activated receptors (PPARs) at the cellular and whole organism levels. Swiss Med Wkly 140: w13071, 2010.

52. Bardot O, Aldridge TC, Latruffe N and Green S: PPAR-RXR heterodimer activates a peroxisome proliferator response element upstream of the bifunctional enzyme gene. Biochem Biophys Res Commun 192: 37-45, 1993.

53. de Gottardi A, Pazienza V, Pugnale P, Bruttin F, Rubbia-Brandt L, Juge-Aubry CE, Meier CA, Hadengue A and Negro F: Peroxisome proliferator-activated receptor-alpha and -gamma mRNA levels are reduced in chronic hepatitis $\mathrm{C}$ with steatosis and genotype 3 infection. Aliment Pharmacol Ther 23: 107-114, 2006.

54. Eslam M, Khattab MA and Harrison SA: Peroxisome proliferator-activated receptors and hepatitis $C$ virus. Therap Adv Gastroenterol 4: 419-431, 2011.

55. Patsouris D, Mandard S, Voshol PJ, Escher P, Tan NS, Havekes LM, Koenig W, März W, Tafuri S, Wahli W, et al: PPARalpha governs glycerol metabolism. J Clin Invest 114: 94-103, 2004.

56. Jiang G, Dallas-Yang Q, Li Z, Szalkowski D, Liu F, Shen X, Wu M, Zhou G, Doebber T, Berger J, et al: Potentiation of insulin signaling in tissues of Zucker obese rats after acute and long-term treatment with PPARgamma agonists. Diabetes 51: 2412-2419, 2002.
57. Dharancy S, Malapel M, Perlemuter G, Roskams T, Cheng Y, Dubuquoy L, Podevin P, Conti F, Canva V, Philippe D, et al: Impaired expression of the peroxisome proliferator-activated receptor alpha during hepatitis $\mathrm{C}$ virus infection. Gastroenterology 128: 334-342, 2005.

58. Clément $S$, Pascarella $S$ and Negro F: Hepatitis $C$ virus infection: Molecular pathways to steatosis, insulin resistance and oxidative stress. Viruses 1: 126-143, 2009.

59. Ozcan U, Cao Q, Yilmaz E, Lee AH, Iwakoshi NN, Ozdelen E, Tuncman G, Görgün C, Glimcher LH and Hotamisligil GS: Endoplasmic reticulum stress links obesity, insulin action, and type 2 diabetes. Science 306: 457-461, 2004.

60. Hotamisligil GS: Role of endoplasmic reticulum stress and c-Jun $\mathrm{NH} 2$-terminal kinase pathways in inflammation and origin of obesity and diabetes. Diabetes 54 (Suppl 2): S73-78, 2005.

61. Christen V, Treves S, Duong FH and Heim MH: Activation of endoplasmic reticulum stress response by hepatitis viruses up-regulates protein phosphatase 2A. Hepatology 46: 558-565, 2007.

62. Wu Z, Jiao P, Huang X, Feng B, Feng Y, Yang S, Hwang P, Du J, Nie Y, Xiao G and Xu H: MAPK phosphatase-3 promotes hepatic gluconeogenesis through dephosphorylation of forkhead box $\mathrm{O} 1$ in mice. J Clin Invest 120: 3901-3911, 2010.

63. Yan L, Lavin VA, Moser LR, Cui Q, Kanies C and Yang E: PP2A regulates the pro-apoptotic activity of FOXO1. J Biol Chem 283: 7411-7420, 2008.

64. Deng X, Zhang W, O-Sullivan I, Williams JB, Dong Q, Park EA, Raghow R, Unterman TG and Elam MB: FoxO1 inhibits sterol regulatory element-binding protein-1c (SREBP-1c) gene expression via transcription factors Sp1 and SREBP-1c. J Biol Chem 287: 20132-20143, 2012.

65. Zhang K, Li L, Qi Y, Zhu X, Gan B, DePinho RA, Averitt T and Guo S: Hepatic suppression of Foxol and Foxo3 causes hypoglycemia and hyperlipidemia in mice. Endocrinology 153: 631-646, 2012

66. Zhang W, Patil S, Chauhan B, Guo S, Powell DR, Le J, Klotsas A, Matika R, Xiao X, Franks R, et al: FoxO1 regulates multiple metabolic pathways in the liver: effects on gluconeogenic, glycolytic, and lipogenic gene expression. J Biol Chem 281: 10105-10117, 2006.

67. Gao TT, Qin ZL, Ren H, Zhao P and Qi ZT: Inhibition of IRS-1 by hepatitis $C$ virus infection leads to insulin resistance in a PTEN-dependent manner. Virol J 12: 12, 2015.

68. Persico M, Masarone M, La Mura V, Persico E, Moschella F, Svelto M, Bruno S and Torella R: Clinical expression of insulin resistance in hepatitis $\mathrm{C}$ and $\mathrm{B}$ virus-related chronic hepatitis: Differences and similarities. World J Gastroenterol 15: 462-466, 2009.

69. Eguchi Y, Mizuta T, Ishibashi E, Kitajima Y, Oza N, Nakashita S, Hara M, Iwane S, Takahashi H, Akiyama T, et al: Hepatitis C virus infection enhances insulin resistance induced by visceral fat accumulation. Liver Int 29: 213-220, 2009.

70. Squillace N, Lapadula G, Torti C, Orlando G, Mandalia S, Nardini G, Beghetto B, Costarelli S and Guaraldi G: Hepatitis C virus antibody-positive patients with HIV infection have a high risk of insulin resistance: A cross-sectional study. HIV Med 9: 151-159, 2008.

71. Moucari R, Asselah T, Cazals-Hatem D, Voitot H, Boyer N, Ripault MP, Sobesky R, Martinot-Peignoux M, Maylin S, Nicolas-Chanoine MH, et al: Insulin resistance in chronic hepatitis C: Association with genotypes 1 and 4, serum HCV RNA level, and liver fibrosis. Gastroenterology 134: 416-423, 2008. 